\title{
THE ROLE OF LASER IRRADIANCE, PULSE REPETITION RATE, AND LIQUID MEDIA IN THE SYNTHESIS OF GOLD NANOPARTICLES BY THE LASER ABLATION METHOD USING AN ND:YAG LASER 1064 NM AT LOW ENERGY
}

\author{
Ali Khumaeni ${ }^{1 *}$, Heri Sutanto ${ }^{1}$, Wahyu Setia Budi ${ }^{1}$ \\ ${ }^{1}$ Department of Physics, Faculty of Science and Mathematics, Diponegoro University, \\ Jl Prof. Soedharto, SH., Tembalang, Semarang 50275, Indonesia
}

(Received: April 2018 / Revised: August 2018 / Accepted: April 2019)

\begin{abstract}
The synthesis of gold nanoparticles with high-purity and narrow size distribution is necessary for applications in the medical field. However, it is difficult to achieve this using chemical methods. In this study, the pulse laser ablation method using an Nd:YAG laser operated at a low-energy of $30 \mathrm{~mJ}$ has been successfully employed to produce gold nanoparticles with the required high purity and narrow size distribution. The role of laser irradiance, laser pulse repetition rate, and liquid media in the characteristics of the nanoparticles produced, such as shape and size distribution, were examined. In the experiment, an Nd:YAG laser beam (1064 $\mathrm{nm}, 7 \mathrm{~ns}$ ) with a low energy of $30 \mathrm{~mJ}$ was irradiated on a high-purity gold plate $(99.95 \%)$ immersed in a liquid medium. The results demonstrate that the average particle diameter and size distribution depended on certain parameters of the laser irradiance, the pulse repetition rate and the liquid medium used in the synthesis process. The diameters of the GNPs increased from 6.5 to $12.3 \mathrm{~nm}$ when the laser irradiance was increased from 12 to $20 \mathrm{GW} / \mathrm{cm}^{2}$. They also increased from 12.3 to $20.7 \mathrm{~nm}$ when the pulse repetition rate was increased from 10 to $15 \mathrm{~Hz}$. In addition, the particle diameters changed in line with different liquid media used; they were much smaller for purified water (diameter of $12.3 \mathrm{~nm}$ ) compared to ethanol (diameter of 15.0 $\mathrm{nm}$ ). However, the shape of the GNPs was the same for these parameters; the GNPs produced by the laser ablation method were spherical. By understanding the effects of these parameters on the characteristics of the GNPs produced by the laser ablation method using a low-energy Nd:YAG laser, GNPs with specific characteristics, namely high purity and narrow size distribution, can be synthesized for specific applications in the medical field.
\end{abstract}

Keywords: GNPs; Gold nanoparticles; Laser ablation method; Laser irradiance; Low energy Nd:YAG laser; Low energy of laser pulse; Pulse repetition rate

\section{INTRODUCTION}

Many scientists and researchers are interested in studying and developing nanoparticles (NPs) for various applications due to their specific characteristics (Tran et al., 2013; Khalil et al., 2017). For example, Adiwibowo et al. (2018) have successfully produced stable $\mathrm{ZnO}$ nanoparticles for detergent applications. NPs have a minute size, with a diameter of 1 to 100 $\mathrm{nm}$. Recently, gold nanoparticles (GNPs) have been produced for certain applications, such as sensors (Raj et al., 2003), photonic devices (Parker \& Townley, 2007), catalysts (Turner et al., 2008), and medical applications (Giljohann et al., 2010). Due to their specific properties,

*Corresponding author's email: khumaeni@fisika.undip.ac.id, Tel. 024 7474754, Fax.024 7474754
Permalink/DOI: https://doi.org/10.14716/ijtech.v10i5.1953 
GNPs have been employed as radiosensitizers, contrast agents, and for drug delivery in the medical field (Dreaden et al., 2012; Cole et al., 2015).

Various methods have been developed for the synthesis of GNPs, such as electrochemical deposition, seeded growth, and vapor phase deposition ( $\mathrm{Rad}$ et al., 2011). However, in chemical methods, additional chemical constituents and stabilizer agents are required during the production process, and therefore the GNPs contain impurities from these. Furthermore, the chemical method produces nanoparticles which have a wide size distribution. Production of high-purity GNPs is necessary for applications in the medical field in order to ensure human health. Therefore, alternative methods for their synthesis are necessary. One such recent method used to produce GNPs is a green process using plants (Aromal et al., 2012).

A physical method based on a pulse laser has also been developed. This technique performs NP synthesis in liquid, producing colloidal NPs (Giorgetti et al., 2012; Semaltios, 2010). The metal NPs produced using this pulse laser ablation (PLA) method have a high purity compared to those produced by conventional methods, such as the chemical method (Al-Azawi \& Bidin, 2015). These NPs are very suitable for specific medical applications which require them to be of high purity, such as when used as contrast agents, for drug delivery, and as radiosensitizers in, for example, cancer and tumor therapy. The PLA method has been applied to the synthesis of silver NPs (AgNPs) and GNPs. Many parameters and variables play important roles in the characteristics of the NPs produced using the PLA method (Abbasi \& Dorranian, 2015), including laser energy, laser pulse repetition rate, and the liquid medium in which they are produced (Al-Nassar et al., 2015).

In this study, a pulse laser ablation method using an Nd:YAG laser operated at a low-energy of $30 \mathrm{~mJ}$ was employed to produce gold nanoparticles in liquid media. The effects of laser pulse repetition rate, laser irradiance, and liquid media in the synthesis of colloidal GNPs using a quiet low-energy Nd:YAG laser were examined to produce nanoparticles with high purity and narrow size distribution. The experimental results show that the gold nanoparticles produced had a much narrower size distribution compared to those produced by the chemical method. Furthermore, the GNPs also had high purity, which is required for applications in the medical field.

\section{EXPERIMENTAL PROCEDURES}

The basic experimental arrangement for the study is shown in Figure 1. A high purity (99.95\%) gold plate was immersed in $10 \mathrm{ml}$ purified water placed in a petri dish (diameter $50 \mathrm{~mm}$ ). A laser beam (Nd:YAG laser, New Polaris II $1064 \mathrm{~nm}$, max energy of $50 \mathrm{~mJ}, 7 \mathrm{~ns}$ ) was irradiated on the gold plate via a convex lens (30 $\mathrm{mm}$ in focal length). In this study, the laser irradiance on the gold plate surface varied between 12 and $20 \mathrm{GW} / \mathrm{cm}^{2}$. The laser pulse repetition rate also varied between 10 and $15 \mathrm{~Hz}$ in order to establish the ablation efficiency of the material. The laser was bombarded on the gold plate for 40 minutes. During the laser irradiation, the gold plate was moved in an XY direction so that the laser always hit new surfaces in order to obtain homogeneous colloidal GNPs.

Various spectroscopic methods were employed for the characterization of the GNPs. The optical properties and surface plasma resonance of the product were characterized by an ultraviolet visible (UV-Vis) absorption spectrometer (Shimadzu 1240 SA). For this purpose, the GNP colloid was placed in a cuvet with dimensions of $12.5 \times 12.5 \times 14 \mathrm{~mm}^{3}$ and inserted in the cell holder. White light was used as a light source. 


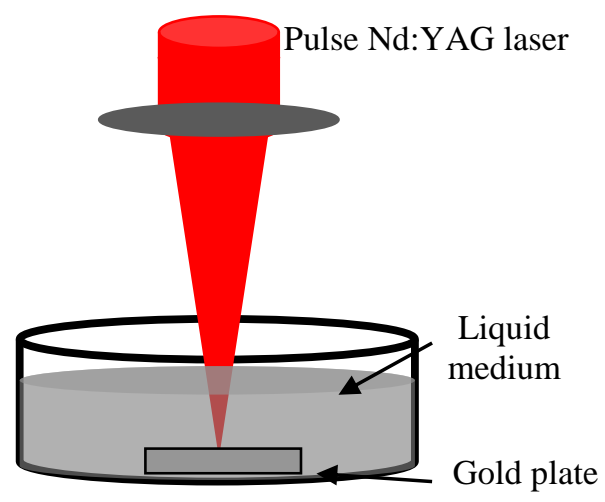

Figure 1 Experimental arrangement used in the study

The morphology of the nanoparticles produced was analyzed using a transmission electron microscope (TEM, JEOL) equipped with an energy dispersive X-ray spectrometer (EDX). TEM samples were prepared in the form of colloidal gold nanoparticles and X-ray diffraction (Rigaku Smartlab, Japan) was used to analyze the crystalline nanoparticles produced. For XRD characterization, the samples were prepared by placing and drying a droplet of the nanoparticle solution on the surface of a silicon wafer. Finally, particle size and size distribution were analyzed using imageJ processing software from the photographs obtained by the TEM.

\section{RESULTS AND DISCUSSION}

\subsection{Role of Laser Irradiance}

First, the role of laser irradiance on the GNP characteristics was examined. Figure 2 shows the absorption spectra of the gold nanoparticles prepared using UV-Vis spectroscopy.

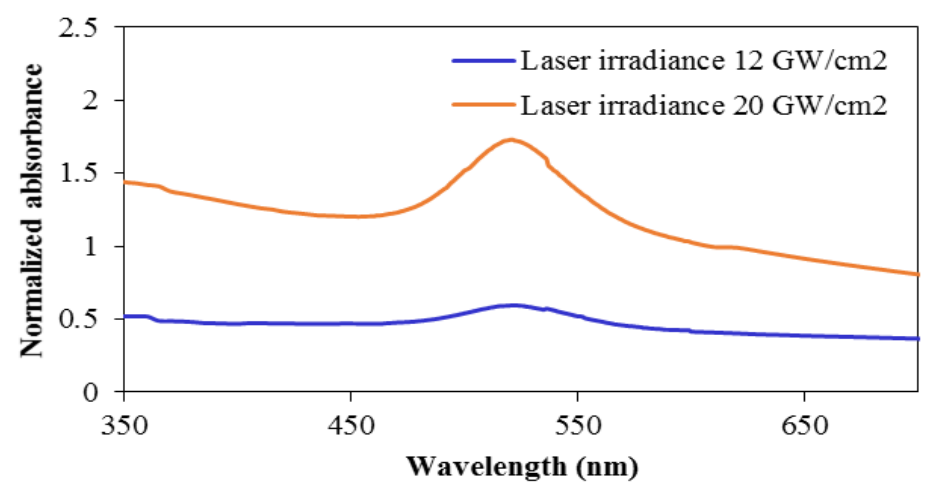

Figure 2 Absorbance spectra of gold nanoparticles synthesized using the pulse laser ablation method with different laser irradiances of $12 \mathrm{GW} / \mathrm{cm}^{2}$ and $20 \mathrm{GW} / \mathrm{cm}^{2}$

Two different laser irradiances were applied during the experiment; the blue and red lines in Fig. 2 represent the spectra with laser irradiance of $12 \mathrm{GW} / \mathrm{cm}^{2}$ and $20 \mathrm{GW} / \mathrm{cm}^{2}$, respectively. The GNPs were prepared using an Nd:YAG laser with a repetition rate of $10 \mathrm{~Hz}$ and laser beam bombardment duration on the surface of the gold of 40 minutes.

The peak of the surface plasmon resonance (SPR) of the GNPs appears clearly for both laser irradiances. The SPR peak occurs at $519 \mathrm{~nm}$ for $12 \mathrm{GW} / \mathrm{cm}^{2}$, and at $521 \mathrm{~nm}$ for $20 \mathrm{GW} / \mathrm{cm}^{2}$. The peak shifts by $2 \mathrm{~nm}$ towards a longer wavelength with the increment in laser irradiance from 12 to $20 \mathrm{GW} / \mathrm{cm}^{2}$, indicating that particle size increases with an increase in laser irradiance. 


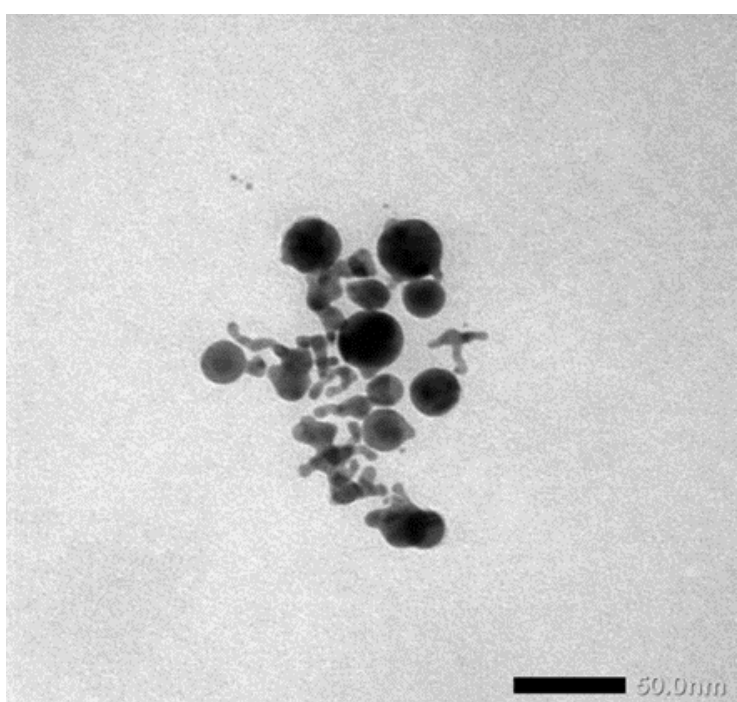

(a)

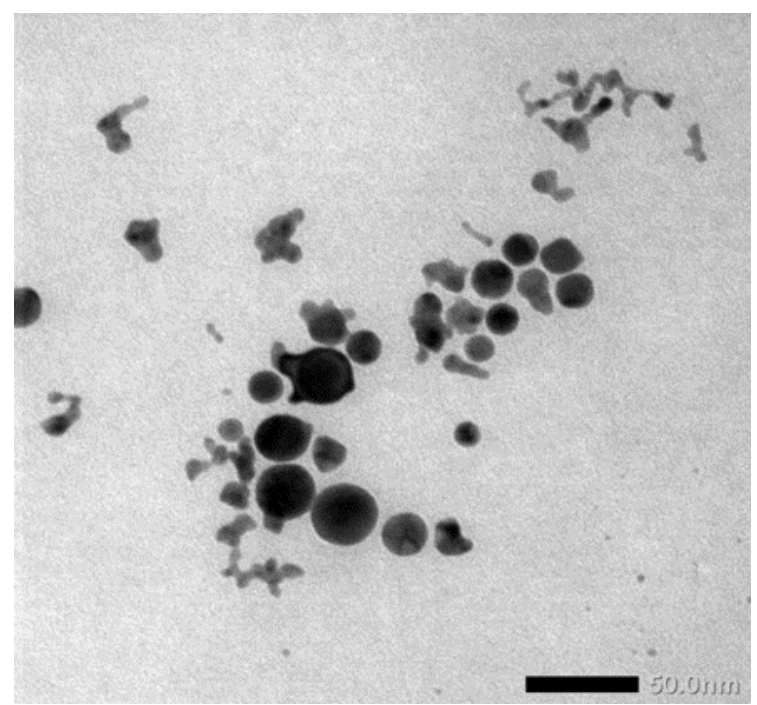

(b)

Figure 3 TEM images of gold nanoparticles and their distribution sizes (inset) obtained by the TEM technique using laser fluencies of: (a) $12 \mathrm{GW} / \mathrm{cm}^{2}$; and (b) $20 \mathrm{GW} / \mathrm{cm}^{2}$

To study the morphology of the GNPs produced, the TEM imaging technique was employed. Figures 3(a) and 3(b) show photographs of the GNPs obtained using the PLA method. All GNPs show a spherical shape with various diameters. This measurement result confirms the indications of nanoparticle shape displayed in Fig. 2, namely that the absorption spectrum has a single SPR peak. This proves that the GNPs were successfully synthesized in a spherical shape.

The distribution of the diameters of the particles obtained using the imageJ processing software derived from the photographs is shown in the inset of Figure 3, with laser irradiances of 12 and $20 \mathrm{GW} / \mathrm{cm}^{2}$, respectively. For $12 \mathrm{GW} / \mathrm{cm}^{2}$ laser irradiance, the average diameter is $6.5 \mathrm{~nm} \pm 3.7$ $\mathrm{nm}$, while in the case of $20 \mathrm{GW} / \mathrm{cm}^{2}$ it is $12.3 \mathrm{~nm} \pm 8.7 \mathrm{~nm}$. This result confirms that increasing the laser irradiance increased the size of the GNPs produced. This is a similar pattern to the results reported by Solati et al. (2013) in the synthesis of silver NPs by the PLA technique using an Nd:YAG laser at fundamental wavelength, namely that the NPs increased in size and had a broader size distribution with increased laser irradiance. It is assumed that the changes in nanoparticle size and size distribution are due to the explosive ejection of material surface by a high-density laser beam. In the case of higher laser irradiance, the distribution of particles ejected from the surface increases (Nichols et al. 2006).

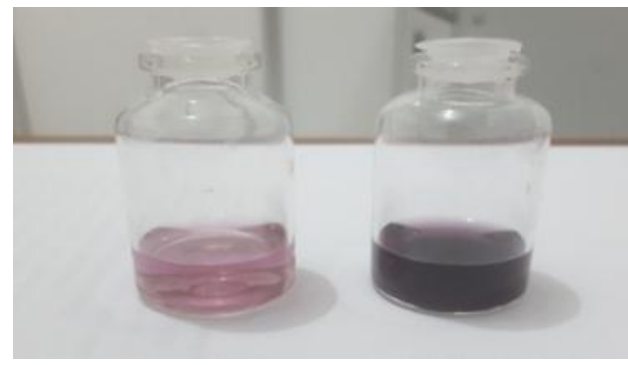

Figure 4 Photographs of colloidal gold nanoparticles produced by laser fluencies of: (a) $12 \mathrm{GW} / \mathrm{cm}^{2}$; and (b) $20 \mathrm{GW} / \mathrm{cm}^{2}$

Figure 4 shows photographs of the GNP colloids produced by the PLA method; Figure 4a shows the GNP product obtained with laser irradiance of $12 \mathrm{GW} / \mathrm{cm}^{2}$, while Figure $4 \mathrm{~b}$ shows that for $20 \mathrm{GW} / \mathrm{cm}^{2}$. The color of the colloids changed from light to dark red with the increase 
in laser irradiance, which indicates that the number of GNPs produced by PLA increases in line with increasing the laser irradiance.

The crystalline gold nanoparticles produced in this study were obtained by an XRD technique and the XRD spectra were displayed in Figure 5. Gold nanoparticles show four specific peaks at $2 \theta=38.1,44.3,64.5$, and 77.7. The peaks corresponded to Bragg reflections (111), (200), (220), and (311) of face center cubic (fcc) lattice. High intensity at 38.1 exhibits that the growth orientation of zero valent gold was fixed in (111) direction. This XRD pattern is specific characteristics of pure gold nanoparticles. From the results, it certified that pure gold nanoparticles have been successfully produced by using this present PLA technique.

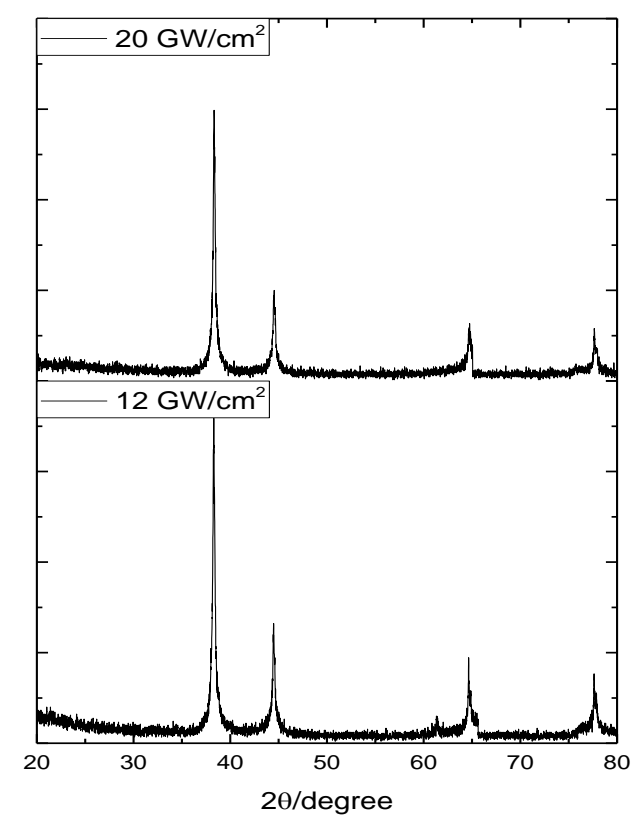

Figure 5 XRD diffractogram from the gold nanoparticles produced by laser irradiance of $12 \mathrm{GW} / \mathrm{cm}^{2}$ and $20 \mathrm{GW} / \mathrm{cm}^{2}$

\subsection{Role of pulse repetition rate}

The role of the pulse repetition rate in the characteristics of the GNPs produced by the PLA technique was studied next. Figure 6 displays the spectra of the GNPs obtained by the UV-Vis spectroscopy technique for laser pulse repetition rates of $10 \mathrm{~Hz}$ (fixed blue line) and $15 \mathrm{~Hz}$ (fixed red line). A single SPR peak clearly occurs for both the10 and $15 \mathrm{~Hz}$ repetition rates, indicating that the GNPs produced by the PLA technique have a spherical shape. The increase in the absorbance values at $15 \mathrm{~Hz}$ indicates that ablation efficiency effectively increases with increasing repetition rate (Xu et al., 2012).

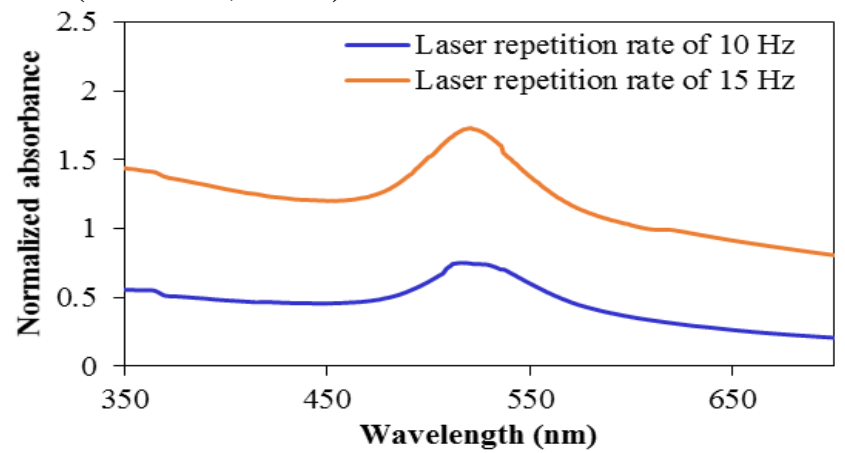

Figure 6 Absorbance spectra of gold nanoparticles produced using the laser ablation method with laser pulse repetition rates of 10 and $15 \mathrm{~Hz}$ 
TEM images of the GNPs produced by the laser ablation method with laser pulse repetition rates of 10 and $15 \mathrm{~Hz}$, including their size distribution, are shown in Figure 7. The laser energy was $30 \mathrm{~mJ}$, and the duration of the laser bombardment onto the pure gold sheet sample was 40 minutes. During the synthesis process, the gold and its container were shaken to obtain homogeneous GNP colloids.

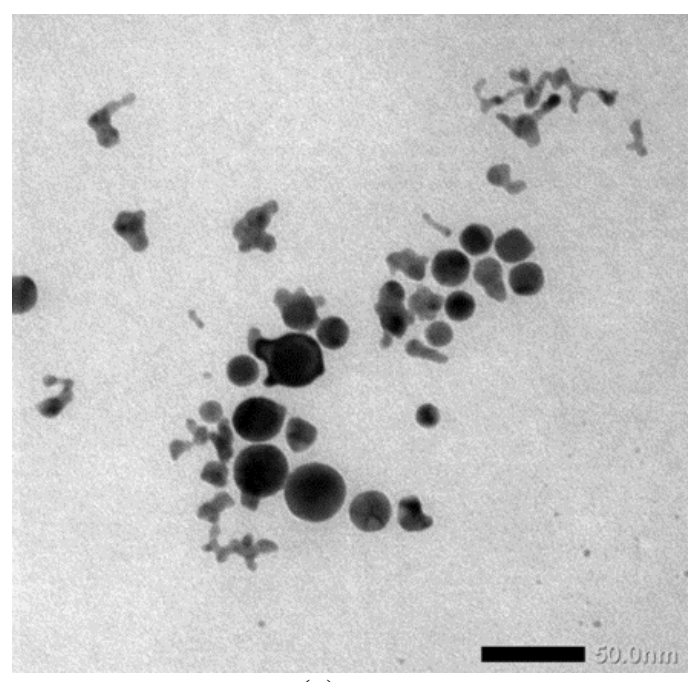

(a)

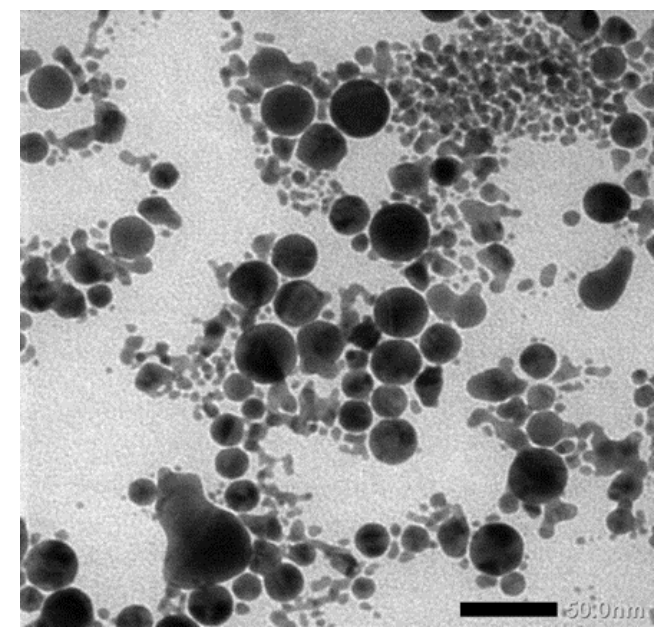

(b)

Figure 7 TEM images of gold nanoparticles and their distribution sizes (inset) obtained by the TEM technique with laser pulse repetition rates of: (a) $10 \mathrm{~Hz}$; and (b) $15 \mathrm{~Hz}$

It can be clearly seen that the shape of the GNPs in both conditions are the same, namely spherical. This is similar to the shape of nanoparticles generally produced by the pulse laser ablation method, as mentioned above. The GNP size distribution is displayed in the inset of each figure. The average diameter and size distribution of the GNPs was obtained using imageJ software at the: (a) $10 \mathrm{~Hz}$ repetition rate; and (b) $15 \mathrm{~Hz}$ repetition rate. The average diameter of the GNPs produced in this study was $12.3 \mathrm{~nm} \pm 8.7 \mathrm{~nm}$ when a laser repetition rate of $10 \mathrm{~Hz}$ was used, as shown in Figure 7a. In the case of a $15 \mathrm{~Hz}$ repetition rate, the average diameter was $20.7 \mathrm{~nm} \pm 9.8 \mathrm{~nm}$, as seen in Figure $7 \mathrm{~b}$. The results confirm that with increasing laser repetition rate, the size of the GNPs produced increases. This result agrees with the work of Zamiri et al. (2013) concerning the synthesis of silver NPs, namely that the diameter of NPs increased with incremental laser energy.

\subsection{Role of Liquid Media}

Finally, the effect of the liquid media on the GNP characteristics was studied; to do this, purified water (aquades) and ethanol were used.

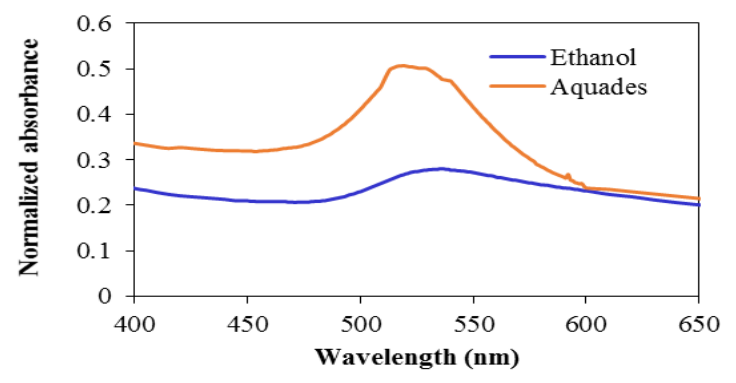

Figure 8 Absorbance spectra of gold nanoparticles produced using the PLA technique with aquades and ethanolliquid media 
Figure 8 displays the absorption spectra obtained from the GNPs produced in the experiment with purified water (red line) and ethanol (blue line). A single peak for SPR appears for both purified water and ethanol, confirming the spherical shape of the GNPs produced with both purified water and ethanol. The absorption intensity for purified water is much higher than that for ethanol, which means that more GNPs are produced in purified water than in ethanol.

To prove that the shapes of the GNPs produced are spherical in the case of purified water and ethanol, images of the GNPs were made using TEM imaging. Figure 9 shows the photographs of the GNPs obtained using the TEM technique. The distribution of GNP size is shown in the inset in each figure. The shape of the GNPs is spherical both for purified water and ethanol. The average diameter of the GNPs produced in purified water was $12.3 \mathrm{~nm} \pm 8.7 \mathrm{~nm}$, as shown in the inset in Fig. 9(a), while in the case of ethanol, the average diameter was $15.0 \mathrm{~nm} \pm 7.5 \mathrm{~nm}$ (inset in Fig. 9b).The average size diameter and size distribution of the particles in purified water were slightly smaller than in the case of ethanol. This might relate to the characteristics of dipole moments in liquid media; purified water has a higher dipole moment than ethanol. As reported by Tilaki et al. (2007), a higher dipole moment of molecules strengthens the bonds between them and the surface of NPs produced. Therefore, the growth mechanism is retarded. The other reason is the low ablation efficiency in ethanol compared to purified water. In the ethanol medium, an ethanol decomposition process takes place during laser ablation, which results in ethanol gas, indicated by numerous gas bubbles produced during laser bombardment. These gases may retard the laser path, which will reduce the laser energy that reaches the target. Lower energy will increase the size of the nanoparticles produced (Moura et al., 2017).

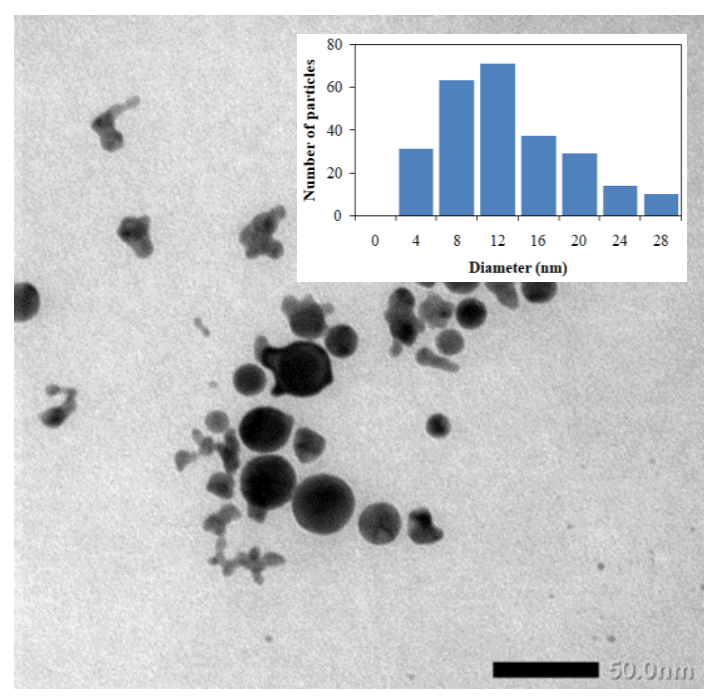

(a)

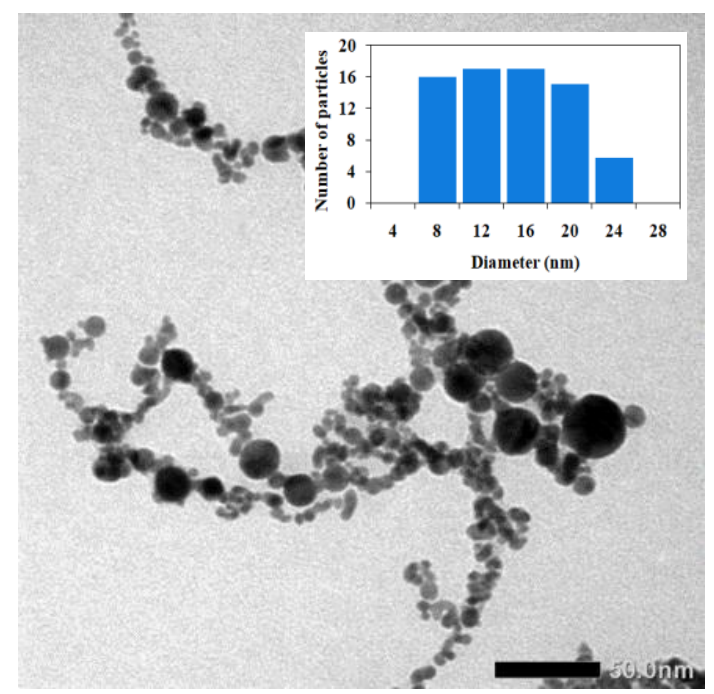

(b)

Figure 9 TEM images of gold nanoparticles and their distribution sizes (inset) obtained by the TEM technique with: (a) purified water; and (b) ethanol liquid media

\section{CONCLUSION}

Gold nanoparticles have been successfully produced in liquid media using the pulse laser ablation method and an Nd:YAG laser operating at a low energy of $30 \mathrm{~mJ}$. The roles of the laser repetition rate, laser irradiance, and liquid media in the characteristics of the GNPs produced by the pulse laser ablation method using an Nd:YAG laser at fundamental wavelength were studied. The results confirm that the laser irradiance affects the average size, size distribution and morphology of the GNPs. Increasing the irradiance from 12 to $20 \mathrm{GW} / \mathrm{cm}^{2}$ resulted in the diameter of the GNPs increasing on average from 6.5 to $12.3 \mathrm{~nm}$. A spherical GNP shape was produced by both laser irradiances. The GNP particle size also increased with increments in the 
pulse repetition rate; particle diameter increased from 12.3 to $20.7 \mathrm{~nm}$ with an increase in repetition rate from $10 \mathrm{~Hz}$ to $15 \mathrm{~Hz}$. When using different liquid media (purified water and ethanol), the diameter of the GNPs produced also changed; the average diameter was $12.3 \mathrm{~nm}$ when using purified water and $15.0 \mathrm{~nm}$ for ethanol. All the GNPs produced in this study had a spherical shape. The results confirm that gold nanoparticles with narrow size distribution and high purity can be successfully produced using the PLA technique. The high-purity GNPs produced have the strong possibility to be applied to the medical field as a contrast agent for cancer diagnosis using CT scanning.

\section{ACKNOWLEDGEMENT}

This study was supported financially by the Ministry of Research, Technology and Higher Education, Indonesia under the Penelitian Terapan Unggulan Perguruan Tinggi project (Contract No. 344-33/UN7.5.1/PP/2017 and 101-122/UN7.P4.3/PP/2018).

\section{REFERENCES}

Abbasi, M., Dorranian, D., 2015. Effect of Laser Fluence on the Characteristics of Al Nanoparticles Produced by Laser Ablation in Deionized Water. Optics and Spectroscopy, Volume 118(3), pp. 472-481

Adiwibowo, M.T., Ibadurrohman, M., Slamet., 2018. Synthesis of ZnO Nanoparticles and Their Nanofluid Stability in the Presence of a Palm Oil-based Primary Alkyl Sulphate Surfactant for Detergent Application. International Journal of Technology, Volume 9(2), pp. 307-316

Al-Azawi, M.A., Bidin, N., 2015. Gold Nanoparticles Synthesized by Laser Ablation in Deionized Water. Chinese Journal of Physics, Volume 53(4), pp. 080803-1-080803-9

Al-Nassar, S.I., Adel, K.M., Mahdi, Z.F., 2015. Study the Effect of Different Liquid Media on the Synthesis of Alumina $\left(\mathrm{Al}_{2} \mathrm{O}_{3}\right)$ Nanoparticle by Pulsed Laser Ablation Technique. Manufacturing Science and Technology, Volume 3(4), pp. 77-81

Aromal, S.A., Vidhu, V.K., Philip, D., 2012. Green Synthesis of Well-dispersed Gold Nanoparticles using Macrotyloma Unflorum. Spectrochimica Acta Part A: Molecular and Biomolecular Spectroscopy, Volume 85(1), pp. 99-104

Cole, L.E., Ross, R.D., Tilley, J.M.R., Vargo-Gogola, T., Roeder, R.K., 2015. Gold Nanoparticles as Contrast Agents in X-Ray Imaging and Computed Tomography. Nanomedicine, Volume 10(2), pp. 321-341

Dreaden, E.C., Alkilany, A.M., Huang, X., Murphy, C.J., El-Sayed, M.A., 2012. The Golden Age: Gold Nanoparticles for Biomedicine. Chemical Society Reviews, Volume 41(7), pp. 2740-2779

Giljohann, D.A., Seferos, D.S., Daniel, W.L., Massich, M.D., Patel, P.C., Mirkin, C.A., 2010. Gold Nanoparticles for Biology and Medicine. Angewandte Chemie International Edition, Volume 49(19), pp. 3280-3294

Giorgetti, E., Miranda, M.M., Marsilli, P., Scarpellini, D., Giammanco, F.J., 2012. Stable Gold Nanoparticles Obtained in Pure Acetone by Laser Ablation with Different Wavelengths. Journal of Nanoparticle Research, Volume 14(1), pp. 1-13

Khalil, M., Liu, N., Lee, R., 2017. Synthesis and Characterization of Hematite Nanoparticles using Ultrasonic Sonochemistry Method. International Journal of Technology, Volume 8(4), pp. 582-590

Moura, C.G., Pereira, R.S.F., Andritschky, M., Lopes, A.L.B., Grilo, J.P.d.F., Nascimento, R.M.d., Silva, F.S., 2017. Effects of Laser Fluence and Liquid Media on Preparation of Small Ag Nanoparticles by Laser Ablation in Liquid. Optics and Laser Technology, Volume 97, pp. 20-28 
Nichols, W.T., Sasaki, T., Koshizaki, N., 2006. Laser Ablation of a Platinum Target in Water. I. Ablation Mechanisms. Journal of Applied Physics, Volume 100(11), pp. 114911-114917

Parker, A.R., Townley, H.E., 2007. Biomimetics of Photonic Nanostructures. Nature Nanotechnology, Volume 2, pp. 347-353

Rad, A.G., Abbasi, H., Afzali, M.H., 2011. Gold Nanoparticles: Synthesising, Characterizing and Reviewing Novel Application in Recent Years. Physics Procedia, Volume 22, pp. 203-208

Raj, C.R., Okajima, T., Ohsaka, T.J., 2003. Gold Nanoparticle Arrays for the Voltammetric Sensing of Dopamine. Journal of Electroanalytical Chemistry, Volume 543, pp. 127-133

Semaltios, N.G., 2010. Nanoparticles by Laser Ablation. Critical Reviews in Solid State and Materials Sciences, Volume 35, pp. 105-124

Solati, E., Mashayekh, M., Dorranian, D., 2013. Effects of Laser Pulse Wavelength and Laser Irradiance on the Characteristics of Silver Nanoparticle Generated by Laser Ablation. Applied Physics A, Volume 112(3), pp. 689-694

Tilaki, R.M., Zad, A.I., Mahdavi, S.M., 2007. Size, Composition and Optical Properties of Copper Nanoparticles Prepared by Laser Ablation in Liquids. Applied Physics A, Volume 88(2), pp. 415-419

Tran, Q.H., Nguyen, V.G., Le, A.T., 2013. Silver Nanoparticles: Synthesis, Properties, Toxicology, Applications and Perspectives. Advances in Natural Sciences: Nanoscience and Nanotechnology, Volume 4(3), pp. 1-20

Turner, M., Golovko, V.B., Vaughan, O.P.H., Abdulkin, P., Berenguer-Murcia, A., Tikhov, M.S., Johnson, B.F.G., Lambert, R.M., 2008. Selective Oxidation with Dioxygen by Gold Nanoparticle Catalysts Derived from 55-Atom Clusters. Nature International Journal of Science, Volume 454, pp. 981-983

Xu, B., Song, R-G., Wang, C., He, W.Z., 2012. Effect of Laser Repetition Rate on Silver Nanoparticles Colloid. Advanced Materials Research, Volume 538, pp. 1888-1891

Zamiri, R., Zakaria, A., Ahangar, H.A., Darroudi, M., Zamiri, G., Rizwan, Z., Drummen, G.P.C., 2013. The Effect of Laser Repetition Rate on the Lasis Synthesis of Biocompatible Silver Nanoparticles in Aqueous Solution Starch. International Journal of Nanomedicine, Volume 8, pp. 233-244 